\section{Formal and semantic similarity of stimuli and recall time*}

\author{
WILLARD N. RUNQUIST \\ University of Alberta, Edmonton 7, Alta., Canada
}

Seven study-test trials were given on paired-associate lists varying in intralist stimulus similarity. Stimuli rhymed, were from the same conceptual category, or were dissimilar. Study time was constant at $2 \mathrm{sec} / \mathrm{item}$, but time allowed for recall on paced test trials was either 1 or $3 \mathrm{sec}$. At the faster rate, the formally similar stimuli produced more interference than did the semantically similar stimuli. There was also some evidence that semantic similarity did not produce much interference early in learning. At the slower rates, the two high-similarity conditions produced substantial but equal amounts of interference. The results are consistent with a sequential coding hypothesis.

This experiment compares the effects of formal and semantic similarity among stimulus terms on paired-associate learning. At the theoretical level, it is based on some commonly held hypothees concerning the processing of stimuli in associative recall. Although these hypotheses take several different specific forms, the basic assumption is that the stimulus, when presented as a cue for recall, undergoes a series of transformations or encodings. The to-be-recalled item exists in the memory system, along with some cue code or codes. Recall depends on the activation of the critical retrieval codes.

The focus in the present investigation is the proposed serial arousal of codes at recall. A representational code results from the direct perception of the stimulus and contains only information concerning its physical attributes. The second coding aroused may be called the elaborative code (Runquist, 1972), and may take several different forms, ranging from letter selection (Postman $\&$ Greenbloom, 1967) to imagery (Paivio, 1971).

Interference resulting from formal similarity among stimuli should primarily be due to the activation of similar or identical representational codes, while interference resulting from semantic similarity should be due to the activation of similar or identical elaborative codes. Taken in conjunction with the assumption that representational codes temporally precede the activation of elaborative codes, this analysis implies that restricting the time allowed for activating retrieval codes should have different effects on the recall of items associated with formally or semantically similar stimuli.

*Supported by Grant APA-88 from the National Research Council of Canada. Marva Blackmore supervised the data collection. Es were Adele James, Marion Shemeluk, and Annabel Lutzer.
Basically, reducing the time allotted for recall should make the activation of semantic elaborative codes more difficult. When formal similarity among word stimuli is high, it is likely that interference is resolved by the use of these semantic codes (Runquist, 1970,1971 ). A c cordingly, interference should increase with restrictions on recall time.

The situation with respect to semantic similarity is somewhat ambiguous. Semantic codes can be highly variable, and it is by no means certain just how interference resulting from semantic similarity is resolved. Nevertheless, it could be argued that restricting recall time with semantically similar stimuli might actually reduce the overall interference by making semantic elaboration more difficult and hence removing some of the interference from this source.

\section{DESIGN AND SUBJECTS}

The experiment involved six groups of Ss, each learning a single paired associate list by the study-test procedure. The design was essentially a 3 by 2 factorial arrangement of three conditions of intralist stimulus similarity (low, high formal, high semantic) orthogonal to two time intervals on the paced test trial which succeeded each study trial ( 1 and $3 \mathrm{sec}$ ).

The Ss were 144 introductory psychology students who volunteered as part of a course requirement. They were assigned to conditions in order of appearance at the laboratory according to a scheme which randomized the order of conditions within blocks containing all six conditions. Six different lists were used within each of the similarity conditions, with four Ss learning each list. The lists were used consecutively, however, thus confounding the nested lists factor with stage of the experiment.

\section{MATERIALS}

The lists each consisted of eight one-syllable nouns paired with two-syllable adjectives. The stimuli for the six lists in each category were arbitrarily chosen by the author from an exhaustive list of common one-syllable words. Both concrete and abstract nouns were included. The stimuli within each list of high formal similarity were rhymes, although spelling sometimes differed. The stimuli rhymed with drum, beer, gate, queen, light, and broom in the six lists. The stimuli for semantically similar lists were related by superordinate category. The six categories were animals, alcoholic beverages, weather phenomena, trees, emotions, and things related to combustion. The stimuli for low-similarity lists were selected to avoid obvious meaningful or phonemic relations.

Five different sets of adjectives were used as response terms. Two sets of lists shared one set of response terms, while the remaining four sets of responses were each assigned to one set of stimuli in each similarity condition. Thus, stimulus and response sets are confounded within the lists factor. The different lists simply served to increase the generality of the results.

\section{PROCEDURE}

Each $S$ received seven study-test sequences. The presentation rate on study trials was $3 \mathrm{sec}$. The time between study and test and test and study was approximately $1.5 \mathrm{sec}$. Four orders of presentation were used on study trials and four different orders were used on test trials. All data were collected by carefully trained undergraduate Es.

Several performance measures obtained from the seven test trials are presented in Table 1. The first column shows the mean total correct responses summed over the seven test trials. At both test times, the two similarity groups performed more poorly than the low-similarity group. While there is little difference between the semantic and formal similarity conditions at $3 \mathrm{sec}$, formal similarity produces more serious disruption in performance with a 1 -sec limit on recall. The overall analysis of variance, however, revealed significant effects only for time $[\mathrm{F}(1,108)=96.86]$ and similarity $[F(2,108)=13.45]$, both $p<.001$. The critical interaction of Time by Similarity resulted in $\mathrm{F}(2,108)=2.03$, which failed to reach the level of 3.07 necessary to establish the reliability of the result.

The second column of Table 1 reports the mean number of trials per item necessary to produce the first correct recall. The same trend apparent in overall performance is also visible in this measure which generally reflects processes operating early in 
Table 1

Summary of Performance on Recall Trials

\begin{tabular}{lcccc}
\hline & $\begin{array}{c}\text { Mean Total } \\
\text { Correct }\end{array}$ & $\begin{array}{c}\text { Trials to } \\
\text { First Correct }\end{array}$ & $\begin{array}{c}\text { Overt } \\
\text { Errors }\end{array}$ & $\begin{array}{c}\text { Error } \\
\text { Ratio }\end{array}$ \\
\hline 3-Sec & & & & .31 \\
Low & 45.0 & 1.2 & 5.8 & .30 \\
Formal & 37.1 & 1.9 & 7.2 & .34 \\
Semantic & 35.0 & 2.1 & 1.6 & .06 \\
1-Sec & 28.9 & 2.9 & 3.0 & .08 \\
Low & 17.4 & 4.9 & 2.8 & .09 \\
Formal & 23.2 & 3.6 & .78 & - \\
Semantic & 3.9 & .07 & & \\
s & & & & \\
\hline
\end{tabular}

acquisition. Moreover, the analysis of variance resulted in a significant Time by Similarity interaction $[F(2,108)=$ $5.43, \mathrm{p}<.01]$ as well as main effects of time $[F(1,108)=88.4 i]$ and similarity $[\mathrm{F}(2,108)=12.35]$. Lists nested within similarity conditions also produced significant variance $[\mathrm{F}(15,108)=2.16, \quad \mathrm{p}<.05$. The nature of the interaction may best be seen by comparing the formally similar and semantically similar groups with the low-similarity group at each time interval. Looking at the interaction one way, the formal similarity group performs more poorly than the semantic similarity group at $1 \mathrm{sec}$, while the reverse is true at $3 \mathrm{sec}$. Viewed another way, the difference between the formal similarity group and the low-similarity group is larger at $1 \mathrm{sec}$, but the difference between the semantically similar group and the low-similarity group is larger at $3 \mathrm{sec}$.

The overt error data also presented in Table 1 were singularly unrevealing. While the high-similarity conditions generally produced more overt errors, the ratio of overt errors to opportunities (overt errors plus omissions) was constant among similarity groups. The incidence of overt errors was extremely low at the 1 -sec test rate. No significance tests were performed on these data.

DISCUSSION

While not overwhelming, the data are consistent with assumptions concerning the coding of stimuli advanced earlier in this paper. Early in acquisition, interference from formally similar (rhyming) stimuli is increased when greater restrictions are placed on the time allotted for recall. The effect of these restrictions when stimuli are semantically similar may be seen to be much less dramatic and might even result in less interference.

There are several points to be made about these results. The first is methodological. Obviously, one cannot equate the amount of similarity between formally and semantically similar sets of stimuli except in the trivial sense of equal ratings (Runquist, 1968). Thus, could these results not simply be due to more "similarity" among formally similar than among semantically similar stimuli? The slower rate is simply not sensitive to these differences, which become manifest when severe recall restrictions are imposed. The critical feature of this argument is that the semantic-formal distinction is not critical to the interaction. Ignoring the fact that it does not state just what the critical variable is, the argument may be countered by pointing out that considerable interference is present at the slower rate with both semantically and formally similar stimuli. In fact, the semantic group performed somewhat more poorly. Since the groups are virtually equivalent at $3 \mathrm{sec}$, even if this is a fortunate happenstance, it does not seem reasonable to assert that somehow the formally similar group still possessed more "interference potential."

The second point to be made is theoretical. The major assumption with which we have been concerned is that representational and elaborative (semantic) codes are activated in sequence. While the obtained interaction is consistent with that hypothesis, other possibilities are not contraindicated. For example, the two codes may be activated in parallel, with representational codes being formed more rapidly. Alternately, there may be a single multiattribute code in which various attributes become accessible at different rates. There is some evidence of this in the fact that formal similarity still produces interference after many trials, despite the fact that sufficient time is available to generate distinctive semantic codes.

The third point to be made refers directly to the results of this experiment. The interaction between formal and semantic similarity only appeared early in the learning sequence and, while reflected in overall performance, was significant only with trials to the first correct response. Inspection of the trial-by-trial recall revealed that on the early trials, the semantic similarity group performed no more poorly than the control under $1-\mathrm{sec}$ recall restrictions, then showed increasing interference as learning proceeded, while the formal similarity group showed interference throughout. These results make it clear that the same processes are not operating throughout the course of learning. Attempting to describe these changes is, of course, speculative, but it may indicate that initially $S$ is unable to generate semantic codes under the severe time restrictions but that further practice allows the codes to be activated, even when they result in interference. Perhaps, $\mathrm{S}$ cannot help but code meaningful material meaningfully, even when it is not to his advantage to do so.

\section{REFERENCES}

PAIVIO, A. Imagery and verbal processes, New York: Holt, Rinehart \& Winston, 1971.

POSTMAN, L., \& GREENBLOOM, $R$. Conditions of cue selection in the acquisition of paired associate lists. Journal of Experimental Psychology, 1967, 73, 91-100.

RUNQUIST, W. N. Rated similarity of high $m$ CVC trigrams and words and low $m$ CCC trigrams. Journal of Verbal Learning \& Verbal Behavior, 1968, 7, 967-968.

RUNQUIST, W. N. Structural effects of letter identity among stimuli in paired-associate learning. Journal of Experimental Psychology, 1970, 84, 152-163.

RUNQUIST, W. N. Stimulus coding and intralist interference. Journal of Experimental Psychology, 1971, 87, 373-377

RUNQUIST, W. N. Intralist interference and stimulus similarity. In C. P. Duncan, L. Sechrest, and A. W. Melton (Eds.), Human memory: Festschrift for Benton $J$. Underwood. New York: Appleton-Century-Crofts, 1972. 